\title{
A new method for filtering speckle noise in vibration signals measured by Laser Doppler Vibrometry for on-line quality control.
}

\author{
Cristina Cristalli, Barbara Torcianti, Jiří Vass (*) \\ AEA srl. Via Fiume 1660030 Angeli di Rosora Italy e-mail: c.cristalli@loccioni.com \\ (*) Department of Circuit Theory, Faculty of Electrical Engineering, Czech Technical University \\ Technická 216627 Prague, Czech Republic e-mail: vassj@,fel.cvut.cz
}

\begin{abstract}
Mechanical fault diagnostics for quality control of manufacturing appliances is often based on the analysis of machine vibrations. In the presence of mechanical faults, vibration signals comprise periodic impulses with a characteristic frequency corresponding to a particular defect.

Vibrometers based on LDV (Laser Doppler Vibrometry) are an alternative to the traditional use of piezo-electric accelerometers, devices that are the most common and popular vibration transducers. Laser vibrometry is now an established technique for vibration measurements in industrial applications where non-contact operations are essential. Despite the advantages of LDV, speckle noise occurs when rough surfaces are measured and the object is moving. Therefore, removal of speckle noise is a crucial point of a reliable system for diagnostics of mechanical faults.
\end{abstract}

This paper deals with the analysis of vibration signals measured by a Laser Doppler Vibrometer from different electromechanical components such as compressors and electrical motors. The goal is to suppress the speckle-noise effect, coming both from the surface of the electro-mechanical components and its movement, in order to perform an automatic test for mechanical fault detection in the production line.

First, data acquisition and its problems are introduced. Then, the chosen solution is presented. In particular, a statistical approach based on kurtosis is used for detection of speckle noise and selection of an undistorted region within the signal. The algorithm is composed of band-pass filtering, segmentation of the signal and computation of a scalar indicator $K R$ (Kurtosis Ratio) for each signal segment, in order to detect outlying samples caused by speckle noise.

Finally, real examples are shown to test the proposed method, and a tool for validation of signal databases is briefly presented.

Key words: Industrial Diagnostics, Laser Doppler Vibrometry, vibration analysis, speckle noise, kurtosis, Quality Control.

Seventh International Conference on Vibration Measurements by Laser Techniques: Advances and Applications, edited by Enrico Primo Tomasini, Proc. of SPIE Vol. 6345, 63450Y, (2006) · 0277-786X/06/\$15 · doi: 10.1117/12.693104 


\section{INTRODUCTION}

Vibration measurements are widely used in industry for detection of mechanical faults. As the faults may arise during the manufacturing process, vibration-based diagnostics is important for quality control of commercial products, such as household appliances. Therefore, extensive research [1] has been conducted to analyze critical mechanical components, such as bearings.

Among the vibro-acoustic sensors, accelerometers are the most frequently employed instruments for mechanical diagnostics. However, Rodriguez et al. [2] revealed several disadvantages of accelerometers, such as problematic installation during quality control in production lines. For this reason, vibrometers based on LDV (Laser Doppler Vibrometry) are particularly attractive for on-line quality control [2-4] owing to the non-contact nature of lasers. However, despite the advantages of LDV, vibration measurements on rough surfaces can be distorted by undesired surface effects, such as the speckle noise.

This paper presents a new method for avoidance of speckle noise in LDV signals. The goal of the proposed method is either confirmation that the measured signal is undistorted, or selection of an unaffected signal portion when errors are present. The method is applied on LDV signals prior to the common diagnostic tasks, such as signal pre-processing, feature extraction and fault classification.

\section{METHOD}

\subsection{Detection of speckle noise}

As it has been assumed that speckle noise appears randomly and unpredictably, a statistical approach for detection of this error has been utilized. In general, many statistical methods investigate properties of the probability density function (PDF) of a vibration signal. However, instead of directly examining the PDF curve, it is generally more informative to calculate a suitable scalar indicator describing the shape of a distribution [1]. Among various shape indicators, kurtosis has been established as one of the most frequently used parameters in diagnostics (e.g. [1,5]).

Kurtosis is defined as the fourth central statistical moment normalized by the fourth power of the standard deviation:

$$
\beta_{2}(x)=\frac{E\left[(x-\mu)^{4}\right]}{\sigma^{4}} .
$$

Kurtosis can generally be used to indicate whether a data set contains impulsive components. It should be emphasized that kurtosis has been employed only for localization of random impulses caused by speckle noise, i.e. detection of periodic impulses related to mechanical defects is not concerned in this paper.

It has been observed that speckle noise has a character of random impulsive noise which contributes to generation of samples with extreme (outlying) amplitudes. Therefore, it can be assumed that vibration signals containing outlying values have been affected by this measurement error. For this reason, a specific indicator has been designed in order to express the amount of outliers in a signal. This indicator has been termed kurtosis ratio $(K R)$ and is defined as:

$$
K R(x)=\frac{\beta_{2}(x)}{\beta_{2}\left(x_{t}\right)},
$$

where kurtosis $\beta_{2}(x)$ is computed from the raw vibration signal $x$, and kurtosis $\beta_{2}\left(x_{t}\right)$ is calculated from the trimmed signal $x_{t}$. The trimmed signal is obtained by thresholding the raw signal: 


$$
x_{t}=\left(t h r_{\text {low }}<x[i]<t h r_{\text {high }}\right) \text {, }
$$

where $t h r_{\text {low }}$ and $t h r_{\text {high }}$ is the lower and the upper threshold, respectively. Both thresholds are automatically computed in order to remove certain percentage of the lowest and the highest amplitudes. As a result, the value of $\beta_{2}\left(x_{t}\right)$ represents a robust estimate of kurtosis, since it is unaffected by the presence of potential outliers.

Detection of speckle noise operates as follows. In the case of a well measured signal, the value of $\beta_{2}(x)$ and $\beta_{2}\left(x_{t}\right)$ are comparable and $K R(x)$ is thus approximately equal to one. On the contrary, the value of $K R(x)$ for a distorted signal is much greater than one, since outliers increase only the value of $\beta_{2}(x)$. The signal is regarded as distorted when $K R(x)$ exceeds the threshold of 2 . This value was experimentally adjusted in order to achieve sufficient sensitivity with a minimum number of false alarms.

\subsection{Selection of an undistorted region}

As multiple measurements are very time-consuming, signals distorted by LDV errors cannot be rejected and measured again, since industrial production is a subject to high demands on time efficiency. Therefore, such signals must be analyzed and further processed in order to avoid the speckle noise and obtain a signal region without any undesired components.

An algorithm for validation of LDV signals has been developed. The input of the algorithm is a raw LDV signal; the output corresponds to the chosen undistorted region within the signal (when no errors are detected, the output equals to the input). The algorithm operates only in the time domain and consists of the following three main steps.

\section{a. Band-pass filtering}

A digital band-pass filter is used to separate a specific frequency band of interest. The filter is designed using the Parks-McClellan algorithm [6] in order to obtain a linear-phase FIR (Finite Impulse Response) filter. The band from $5 \mathrm{kHz}$ to $10 \mathrm{kHz}$ was selected to avoid structural resonance frequencies.

\section{b. Segmentation}

Filtered signal is divided into overlapping segments. Theoretically, the length of segments should be maximized, since confidence of statistical indicators increases with an increasing number of data points. On the other hand, segments should not be excessively long as good time resolution is necessary to detect the impulses of short duration. For this reason, $80 \%$ overlap is used in order to fulfill both requirements of accurate time resolution and sufficient segment length for reliable estimation of kurtosis.

\section{c. Kurtosis ratio}

Kurtosis ratio is computed for each segment $s_{i}$ of the filtered signal. If the condition $K R\left(s_{i}\right)<2$ is fulfilled, the analyzed segment is regarded as undistorted. The region comprising the highest number of successive undistorted segments is chosen as the best candidate for the correct portion of the signal. 


\section{RESULTS}

This section provides examples of the signal validation procedure. Measurements were conducted on good (healthy) bearings of electrical motors and therefore no fault-related impulses should be present in the LDV signals.

The first example in Fig. 1 represents a successful case, in which the selected region is undistorted and the region length is satisfactory $(31.6 \%)$. As can be observed, band-pass filtering highlights the impulsive components, although the full-band signal also exhibits several random impulses. Note that computation of STFT (Short-Time Fourier Transform) is not included in the algorithm, it is shown in Fig. 1 for visualization purposes only.

On the contrary, filtering is necessary in the second example, in which the measurement error is considerably less evident. Indeed, the impulses can clearly be seen only in Fig. 2(c), since the wide-band spectrum is dominated by structural resonance frequencies. Selection of an undistorted region is very difficult due to a large number of measurement errors. In fact, only a very short region is available (4.4\%), resulting in a need to slightly shift the position of the laser beam and measure the vibration signal again, or inform the human operator about an excessively small amount of correct data. On the other hand, the selected region indeed remains undistorted as no random impulses can be perceived during the hearing test.
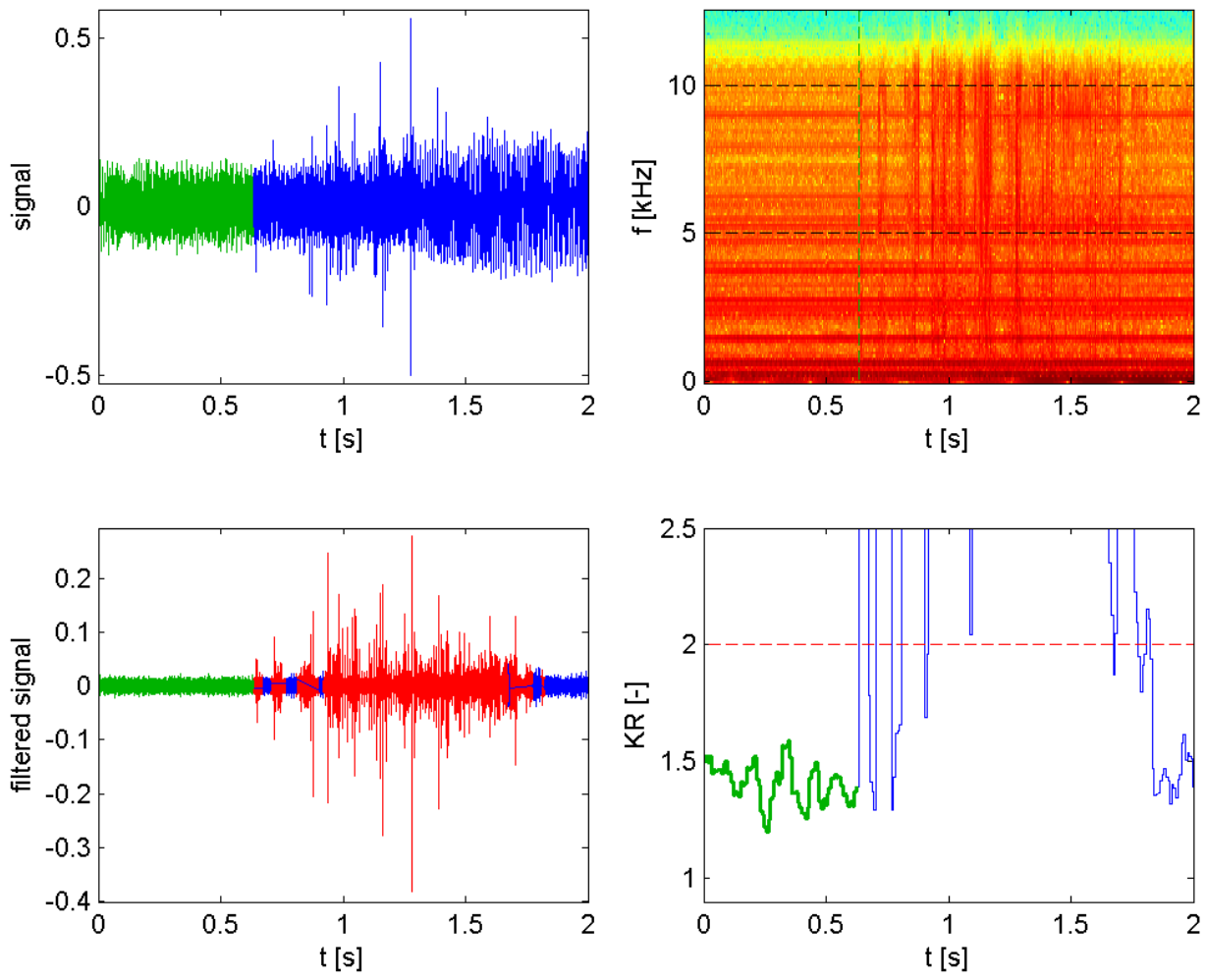

Fig. 1: Successful selection of an undistorted region 

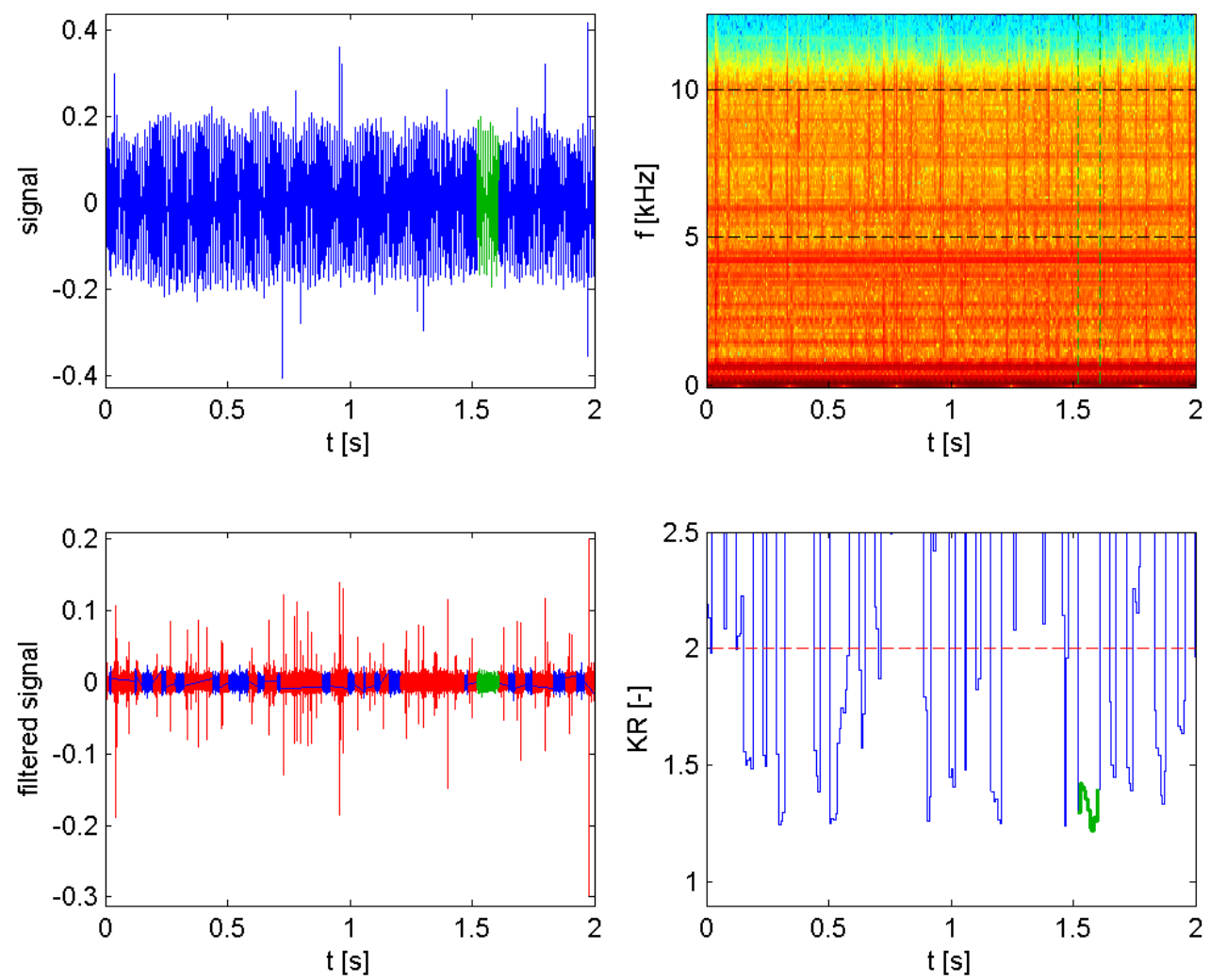

Fig. 2: Selection of an excessively short region

\section{VALIDATION OF SIGNAL DATABASES}

The proposed algorithm can be used for two main purposes: 1) on-line validation of a currently measured LDV signal (prior to detection and classification of mechanical faults); 2) off-line validation of large signal databases. As requirements differ for each of these tasks, we have implemented the on-line system in the LabVIEW environment, whereas the off-line application was created in Matlab. Figure 3 depicts the control panel of our graphical user interface SVT (Signal Verification Tool).

The main advantage of SVT is the ability to automatically process an entire directory of files, where each file corresponds to one measurement. Overall results can be exported to an Excel table, which also contains numerical values of the input parameters, number of good and distorted signals, date and time of analysis and other informative indicators. In addition, detected region is automatically saved for each distorted measurement, and an image is stored for subsequent visual inspection (such as Figures 1 and 2). The tool also provides flexible I/O settings, such as selection of file format (txt or wav), number of output images $(1,2,4)$ and import/export of the algorithm parameters. In summary, SVT is intended to eliminate unnecessary manual labour when analyzing large amount of data, and it has been developed for easy preparation of testing signals for mechanical fault diagnosis. 


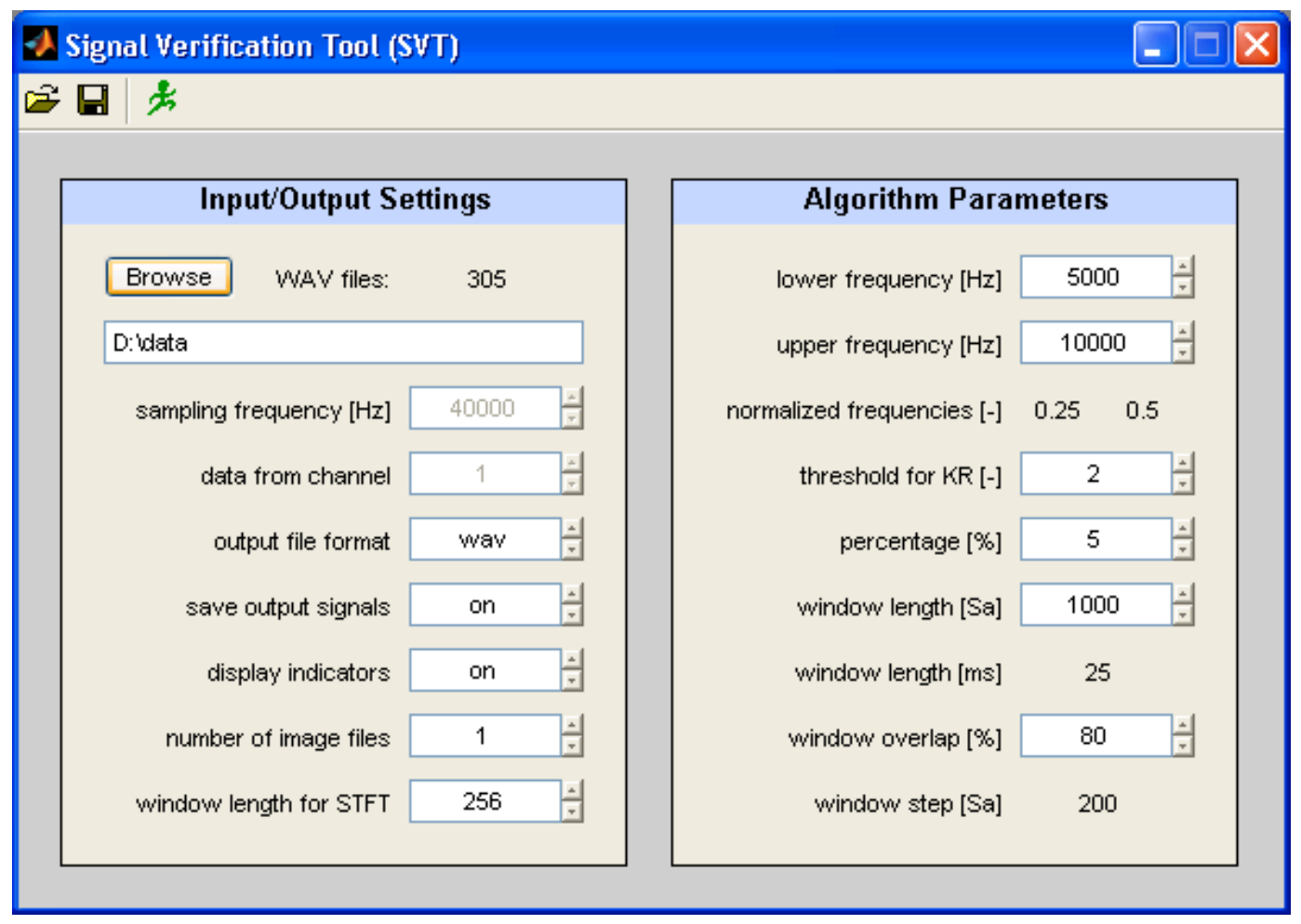

Fig. 3: Graphical user interface for validation of large amount of vibration signals

\section{CONCLUSION}

This paper presents a new method for the validation of vibration signals measured by Laser Doppler Vibrometry (LDV). The method is applied on LDV signals measured on rough surfaces, which are subject to undesired interference effects, such as speckle noise.

Detection of speckle noise is based on a new statistical indicator $K R$ (kurtosis ratio), defined as a ratio of the standard kurtosis and a robust estimate of kurtosis. As a result, $K R$ is able to express the amount of outlying samples in the data, and can thus be effectively used for detection of random impulses generated by speckle noise. As additional measurements are time-consuming, signals affected by speckle noise must be further processed in order to find an undistorted portion of the signal. Straightforward solution of this task has been obtained by computation of $K R$ for individual segments of the analyzed signal. Moreover, sensitivity of $K R$ is significantly improved by band-pass filtering, which enhances the random impulses hidden in the raw LDV signal.

As demonstrated by experimental results, the algorithm is able to effectively avoid the speckle noise generated on rough surfaces of bearings in electrical motors. Therefore, the method can play an important role in LDV-based diagnostic systems, since correctness of measured data should always be ensured prior to subsequent fault detection and classification. Advantages of the proposed method are simplicity, computational efficiency and low requirements on optimization of the input parameters.

Finally, validation of large signal databases is discussed and a graphical user interface SVT (Signal Verification Tool) is briefly presented. 


\section{ACKNOWLEDGEMENTS}

This work has been supported by the research program MSM 6840770014 "Research in the Area of the Prospective Information and Navigation Technologies" and the GA ČR grant No. 102/03/H085 "Biological and Speech Signal Modelling". This research has been supervised by Prof. Ing. Pavel Sovka, CSc., Department of Circuit Theory, FEE CTU and Doc. Ing. Radislav Šmíd, Ph.D., Department of Measurement, FEE CTU. The help of both supervisors is much acknowledged.

\section{REFERENCES}

1. Tandon N. , Choudhury A., "A review of vibration and acoustic measurement methods for the detection of defects in rolling element bearings." Tribology International, Vol. 32, No. 8, Aug. 1999, pp. 469-480.

2. Rodriguez R.M., Cristalli C., Paone N., "Comparative study between laser vibrometer and accelerometer measurements for mechanical fault detection of electric motors." 5th International Conference on Vibration Measurements by Laser Techniques: Advances and Applications, SPIE Proceedings, Vol. 4827, pp. 521-529, 2002.

3. Paone N., Scalise L., Stavrakakis G., Pouliezos A., "Fault detection for quality control of house-hold appliances by non-invasive laser Doppler technique and likelihood classifier.", Measurement, Vol. 25, pp. 237-247, 1999.

4 Vass J., Cristalli C., "Bearing fault detection for on-line quality control of electric motors." Proc. 10th IMEKO TC10 International Conference on Technical Diagnostics, Budapest: Hungarian Academy of Sciences, Vol. 1, pp. 93-97, June 2005, ISBN 963-86586-4-9.

5. Heng R.B.W., Nor M.J.M., "Statistical analysis of sound and vibration signals for monitoring rolling element bearing condition", Applied Acoustics, Vol. 53, No. 1-3, Jan.-Mar. 1998, pp. 211-226.

6. $\quad$ Saramäki T., "Finite Impulse Response Filter Design.” Handbook for Digital Signal Processing. S.K. Mitra and J.F. Kaiser Eds., Wiley-Interscience, N.Y., 1993, Chap. 4. 